\title{
Segurança na aplicação da eletroestimulação neuromuscular no doente crítico: estudo piloto
}

\section{Safety in application of neuromuscular electrical stimulation in critically ill patients: pilot study}

\section{Daniela de Souza Pinto', Helder Brito Duarte ${ }^{2}$, Camila de Almeida Costta ${ }^{3}$, Jorge Luis Motta dos Anjos $^{4}$, Ludmilla Campos Gaspar ${ }^{5}$, Reinaldo Luz Melo ${ }^{6}$, Camilla de Souza Menezes ${ }^{7}$}

'Universidade Salvador, Hospital da Cidade. Salvador, Bahia, Brasil. ORCID: 0000-0001-6789-4369. dani_souzaP@hotmail.com ${ }^{2}$ Autor para correspondência. Universidade Salvador, Hospital da Cidade. Salvador, Bahia, Brasil. ORCID: 0000-0002-4656-750X helderphysio@gmail.com 3Universidade Salvador, Hospital da Cidade. Salvador, Bahia, Brasil. ORCID: 0000-0002-4369-1273. costta_camila@hotmail.com ${ }^{4}$ Hospital Geral Roberto Santos. Salvador, Bahia, Brasil. ORCID: 0000-0003-2897-9858. jorgelmanjos@hotmail.com 5Universidade Salvador, Hospital da Cidade. Salvador, Bahia, Brasil. ORCID: 0000-0002-8100-5502. camposludmilla@yahoo.com.br שUniversidade Salvador, Hospital da Cidade. Salvador, Bahia, Brasil. ORCID: 0000-0003-1250-7186. reinaldoluzmelo@hotmail.com ${ }^{7}$ Fundação Estatal Saúde da Família, Fundação Oswaldo Cruz. Salvador, Bahia, Brasil. ORCID: 0000-0002-4617-1396. millaa_menezes@hotmail.com

RESUMO I INTRODUÇÃO: A Neuromuscular Electrical Stimulation (NMES) é um importante aliado do paciente crítico, favorecendo contrações ativas mesmo em estado que requer imobilidade. OBJETIVO: Verificar a segurança da aplicação da NMES em Unidades de Terapia Intensiva (UTIs) de um hospital público da cidade de Salvador. MÉTODO: Trata-se de estudo piloto, de intervenção. Os dados foram coletados no período de fevereiro a junho de 2018, com amostra por conveniência em uma população de pacientes críticos intubados e em uso de vasopressores. Foi aplicada uma única sessão de 45 minutos de NMES em ambos os quadríceps (músculo reto femoral e vasto lateral), sendo coletados os seguintes dados hemodinâmicos 5 minutos antes da aplicação e logo após a terapêutica: frequência cardíaca, pressão arterial sistólica, diastólica e média; e frequência respiratória. Estes dados foram avaliados seguindo recomendações de segurança já descritas previamente. Para análise estatística, as variáveis foram descritas através de médias e desvio-padrão, mediana e intervalo interquartílico e percentuais obtidos nas variáveis do estudo. A distribuição dos dados foi avaliada pelo teste Shapiro-Wilk, e os testes Mann Whitney e T de student foram utilizados. RESULTADOS: A amostra foi composta por 8 pacientes sendo 1 excluído. Destes, $85,7 \%$ era do sexo feminino, sendo o diagnóstico clínico de Sepse evoluindo para choque em $85,7 \%$, média da idade de $61 \pm 9,5$ anos e APACHE II de $29 \pm 5,5$. Não foram evidenciadas diferenças estatísticas em relação aos dados hemodinâmicos coletados pré e pós eletroestimulação. Estes dados são semelhantes aos resultados encontrados por outros autores em populações sem uso de vasopressores. CONCLUSÃO: É possível sugerir que a aplicação da NMES no doente crítico em uso de vasopressores é uma técnica segura e viável desde que respeitando os limites estabelecidos e parâmetros corretos baseados em evidências.

PALAVRAS-CHAVE: Estimulação elétrica nervosa transcutânea. Metabolismo energético. Mobilização precoce. Unidades de terapia intensiva.
ABSTRACT | INTRODUCTION: Neuromuscular Electrical Stimulation (NMES) is an important ally of the critically ill patient, favoring active contractions even when in a state that requires immobility. OBJECTIVE: The objective of this study was to verify the safety of NMES application in Intensive Care Units (ICUs) of a public hospital in the city of Salvador. MATERIAL AND METHODS: This is a pilot, study of intervention. Data were collected from February to June 2018 , having as population critically ill intubated patients using vasopressors. A single 45-minute NMES session was applied to both quadriceps (rectus femoris and vastus lateralis), and the following hemodynamic data were collected 5 minutes before application and soon after therapy: cardiac frequency, systolic, diastolic and medium blood pressure and respiratory frequency. Data were collected following security recommandations, described earlier. For statistical analysis, the variables were described by mean and standard deviation, median and interquartile interval and percentages obtained for the variables of the study. The distribution of data was evaluated by the Shapiro-Wilk test and Mann Whitney test and t test were used. RESULTS: The sample consisted of 8 patients, being 1 excluded. Of these, $85.7 \%$ were female, with a clinical diagnosis of Sepsis in $85.7 \%$, mean age of $61 \pm 9.5$ years and APACHE II of $29 \pm 5.5$. No statistical differences were observed in relation to hemodynamic data collected before and after electrostimulation. These data are similar to those found by other authors in populations without vasopressors usage. CONCLUSION: It is possible to suggest that the application of NMES in critically ill patients using vasopressors is a safe and viable technique as long as respecting the established limits and correct evidence-based parameters.

KEYWORDS: Transcutaneous electric nerve stimulation. Energy metabolism. Early mobilizatio. Intensive care units. 


\section{Introdução}

O avanço da medicina tem propiciado inúmeros benefícios em relação à sobrevida do doente crítico. Porém, alguns desses pacientes vivenciam efeitos negativos proporcionados por longos períodos de repouso no leito'. Esta situação está associada ao imobilismo que, por sua vez, traz consigo inúmeros efeitos adversos. Dentre eles, a fraqueza muscular generalizada, que é ocasionada não somente pela estase no leito, mas também pela sepse, falência de múltiplos órgãos, inflamação sistêmica, uso de ventilação mecânica prolongada e de medicações como bloqueadores neuromusculares e corticoides ${ }^{1,2}$.

Visto que o doente crítico em enfrentamento de uma situação aguda é incapaz de executar qualquer mobilização ativa, é necessário buscar alternativas eficazes e seguras em promover a redução dessa inércia e minimizar os efeitos do imobilismo ${ }^{3}$. Diante disso, a corrente Neuromuscular Eletrical Stimulation (NMES) se apresenta como alternativa de prevenção precoce da fraqueza muscular adquirida na Unidade de Terapia Intensiva (UTI) principalmente em pacientes graves que necessitam de ventilação mecânica, uso de sedação e medicamentos vasoativos ${ }^{4,5}$.

Os benefícios do uso desta estratégia já foram descritos e comprovados anteriormente por outros pesquisadores. Dentre eles destacam-se: regeneração do nervo periférico, diminuição da atrofia muscular, mudança da conformação da fibra muscular e aumento da atividade de enzimas oxidativas ${ }^{6,7}$. Sendo assim, estes efeitos são alcançados a partir de uma descarga elétrica terapêutica na fibra muscular local, que proporciona muitas vezes a contração da musculatura sem alteração de ângulo articular, gerando uma isometria. Este tipo de contração muscular possui como resultante a alteração da microcirculação do indivíduo, favorecendo obstrução mecânica do fluxo sanguíneo muscular, fazendo com que os metabólitos produzidos durante esta contração se acumulem. Por sua vez, esta produção resulta numa excitação dos quimiorreceptores musculares promovendo um aumento expressivo da atividade nervosa simpática ocasionando elevação da frequência cardíaca e aumento da resistência vascular que está associada à pressão arterial ${ }^{8}$.
Diante do exposto, a respeito dos benefícios da NMES em nível muscular e do seu impacto em nível circulatório, percebe-se a necessidade de comprovar a segurança da aplicação da técnica em pacientes críticos, visto que, esta técnica possui resultados comprovados por estudos anteriores impactando em desfechos referentes a redução do tempo de ventilação mecânica, alta hospitalar precoce e prevenção do desenvolvimento de polineuropatia no paciente crítico $4,5,7,10$.

Portanto, a partir da observação dos efeitos positivos da NMES é importante comprovar a segurança de sua aplicação em pacientes críticos. Sendo assim, o objetivo desse estudo foi verificar a segurança da aplicabilidade da eletroestimulação neuromuscular e definir se existem alterações que impactem substancialmente na hemodinâmica em doentes críticos internados em UTIs de um hospital público da cidade de Salvador.

\section{Metodologia}

Trata-se de um estudo piloto, de intervenção, realizado nas UTIs adulto de um Hospital Público da rede estadual de saúde de Salvador-BA. A amostra foi por conveniência, composta por pacientes internados nestas UTIs que se adequassem aos critérios de inclusão. A coleta foi realizada no período de fevereiro a junho do ano de 2018.

Esta pesquisa foi aprovada pelo Comitê de Ética em Pesquisa do Hospital Geral Roberto Santos, conforme parecer $n^{\circ} 2.437410$ e CAAE $n^{\circ}$ : 80977417.9.0000.5028. Foi garantido o anonimato e o sigilo das informações aos participantes da pesquisa, sendo os familiares instruídos a lerem e ouvir o termo de consentimento livre e esclarecido que trazia informações gerais do estudo e confirmava a participação voluntária do paciente na pesquisa.

Os critérios de inclusão foram estabelecidos: ter participação autorizada pelo familiar ou responsável, ter idade entre 18 e 75 anos; estar em uso de ventilação mecânica invasiva, estar em uso de cateter de pressão arterial invasiva, possuir um Acute Physiology and Chronic Health Evaluation (APACHE) II maior ou igual a 
13; Richmond Agitation-Sedation (RASS) $\leq 0$; Pressão positiva expiratória final (PEEP) $\leq 10 \mathrm{mmHg}$; Fração inspirada de oxigênio $\leq 60 \%$; estar em dose limite e estável por 1 hora antes da intervenção de: Noradrenalina $\leq$ $0,2 \mu \mathrm{g} / \mathrm{kg} / \mathrm{min}$; Dobutamina $\leq 8 \mu \mathrm{g} / \mathrm{kg} / \mathrm{min}$; Inotrópico $\leq 0,25 \mu \mathrm{g} / \mathrm{kg} / \mathrm{min}^{11,12}$.

Foram excluídos os pacientes que possuíam gravidez, morte encefálica, doença neuromuscular preexistente, lúpus eritematoso, obstáculos técnicos para implementação da NMES como fraturas ósseas, malignidade em estágio final, marcapasso, enzimas cardíacas elevadas (CK-MB>16,0 U/L e Troponina I>0,034 $\mathrm{ng} / \mathrm{mL}$ ), realização de hemodiálise no período, ventilação com neuroproteção e dispositivo de mensuração de pressão intracraniana, hemorragia ativa, instabilidade hemodinâmica com Pressão Arterial Média (PAM) fora do intervalo entre 65 e $110 \mathrm{mmHg}$, Pressão Arterial Sistólica (PAS) $>180 \mathrm{mmHg}$ ou $<90$ $\mathrm{mmHg}$, saturação periférica de Oxigênio (SpO2) $\leq 90 \%$; Frequência Respiratória (FR) $>40$ irpm, Frequência Cardíaca (FC)>130 bpm $5,11,13,14$.

\section{Mensurações hemodinâmicas}

Foram mensurados os sinais vitais como: PAS, PAD, PAM, FC, FR através de sistema de monitorização disponibilizado no hospital. Os pacientes tiveram seus sinais vitais avaliados durante todo o procedimento por critério de segurança e estes foram registrados 5 minutos antes e logo após a aplicação do protocolo.

\section{Protocolo de Intervenção}

Foi realizada uma única intervenção de NMES através do aparelho Neurodyn III neuromuscular stimulator lbramed ${ }^{\circledR}$ por 45 minutos em ambos os quadríceps, utilizando 2 eletrodos $(5 \times 5 \mathrm{~cm})$ em cada coxa. Os eletrodos foram posicionados segundo os preceitos da aplicação eletroterapêutica a qual é recomendada a fixação do polo negativo no ponto motor do reto femoral e o polo positivo distalmente na coxa priorizando a junção miotendinosa de forma a otimizar a propagação do estímulo, através da técnica conhecida como "mioenergética", a qual coloca-se um eletrodo no inicio do ventre muscular e outro do mesmo canal mais distal, nesse mesmo músculo ${ }^{4,5}$.
Os parâmetros foram empregados nos valores já pesquisados por outros autores ${ }^{4,5}$ : frequência de $50 \mathrm{~Hz}$, largura de pulso de $400 \mu \mathrm{s}$, tempo ativo de 6 segundos e 12 segundos de tempo de descanso, a intensidade foi ajustada de acordo com a contração muscular visível ou palpável nos músculos citados.

\section{Critérios de Interrupção do protocolo}

O protocolo de intervenção seria interrompido caso houvesse eventos importantes como: redução de $4 \%$ da SpO2 inicial; aumento ou diminuição de $20 \%$ da PAM ou sair do intervalo entre 65 e $110 \mathrm{mmHg}$, $P A S>180 \mathrm{mmHg}$; FC>130 bpm e FR>40 $\mathrm{irpm}^{13,14}$. Caso acontecesse, toda a equipe seria acionada visando a segurança do paciente e sua estabilidade.

\section{Análise estatística}

As variáveis contínuas colhidas de forma individual foram agrupadas em média, desvio padrão, mediana e intervalo interquartil, após isso, foram divididos em instantes pré e pós eletroestimulação e compiladas em uma tabela do Microsoft Excel. Após isso, os dados foram transferidos para o software Bioestat 5.3, onde as variáveis foram expressas em média e desvio padrão, nos casos em que a distribuição dos dados foi normal, mediana e intervalo interquartílico para variáveis com distribuição assimétrica e medidas de frequência para variáveis nominais.

Para comparação dos resultados antes e após a NMES, inicialmente foi avaliada a distribuição dos dados através do teste de Shapiro Wilk e após essa verificação foram utilizados os testes $T$ Student, para variáveis com distribuição simétrica ou Mann-Whitney para as variáveis com distribuição assimétrica, sendo definida a significância estatística através de valores de $p<0,05$.

\section{Resultados}

Foram recrutados 8 pacientes para a pesquisa, porém 1 foi excluído por não apresentar contração visível ou palpável. A maior distribuição dos doentes foi do sexo feminino $85,7 \%$, com diagnóstico clínico $(85,7 \%)$ em sua totalidade por Sepse evoluindo para choque, média de $61 \pm 9,5$ anos de idade e com Apache de 29 \pm 5 ,5. Conforme dados descritos na Tabela 1. Todos os pacientes estiveram em uso de Noradrenalina com critérios seguros para a mobilização. 
Tabela 1. Perfil da população de acordo com as características gerais. Salvador/BA, 2018

\begin{tabular}{lcr}
\hline Variáveis & $\mathbf{n = 7}$ & \\
\hline Sexo feminino (\%) & 6 & 85,7 \\
Diagnóstico & & \\
Clínico (\%) & 6 & 85,7 \\
Cirúrgico (\%) & 1 & 14,3 \\
Idade, média \pm DP & & $61 \pm 9,5$ \\
APACHE II, média \pm DP & & $29 \pm 5,5$ \\
\hline
\end{tabular}

Legenda: DP: Desvio Padrão; APACHE: Acute Physiology and Chronic Health Evaluation.

A Tabela 2 evidencia a descrição geral dos dados pré e pós eletroestimulação. É possível notar através desta distribuição que os pacientes estavam em um limiar considerado seguro para a realização da terapêutica, não havendo alterações importantes para suspender o protocolo.

Tabela 2. Distribuição individual dos dados das variáveis de cada paciente pré e pós eletroestimulação. Salvador/BA, 2018

\begin{tabular}{ccccccccccc}
\hline Pacientes & \multicolumn{2}{c}{ FC } & \multicolumn{2}{c}{ PAS } & \multicolumn{2}{c}{ PAD } & \multicolumn{2}{c}{ PAM } & \multicolumn{2}{c}{ FR } \\
\hline & Pré & Pós & Pré & Pós & Pré & Pós & Pré & Pós & Pré & Pós \\
P1 & 105 & 106 & 116 & 135 & 59 & 72 & 78 & 93 & 18 & 24 \\
P2 & 70 & 77 & 177 & 188 & 52 & 58 & 94 & 101 & 21 & 20 \\
P3 & 77 & 79 & 120 & 118 & 60 & 60 & 80 & 79 & 18 & 18 \\
P4 & 102 & 103 & 108 & 119 & 68 & 67 & 81 & 84 & 18 & 18 \\
P5 & 96 & 98 & 125 & 122 & 74 & 72 & 91 & 89 & 17 & 17 \\
P6 & 97 & 95 & 119 & 118 & 41 & 39 & 67 & 65 & 16 & 18 \\
P7 & 105 & 95 & 107 & 96 & 62 & 60 & 77 & 72 & 30 & 31
\end{tabular}

Legenda: FC: Frequência Cardíaca; PAS: Pressão Arterial Sistólica; PAD: Pressão Arterial Diastólica; PAM: Pressão Arterial Média; FR: Frequência Respiratória

A Tabela 3 aponta valores de média, desvio padrão, mediana e intervalo interquartil, bem como os testes comparativos utilizados para cada variável seguindo os preceitos do teste de verificação de Shapiro Wilk. Nesta tabela é visto valores hemodinâmicos com discretas variações para mais ou para menos, porém sem atingir a significância estatística $(p>0,05)$.

Tabela 3. Comparação antes e depois da variáveis hemodinâmicas - Salvador/BA, 2018

\begin{tabular}{lcccc}
\hline Variáveis & \multicolumn{2}{c}{ Pré } & \multicolumn{2}{c}{ Pós } \\
\hline & Média \pm DP & Mediana (IIQ) & Média \pm DP & Mediana (IIQ) \\
FC & & $97(86,5-103,5)$ & $95(87-100,5)$ & $0,89 *$ \\
PAD & $60(55,5-65)$ & & $60(59-69,5)$ & $0,84 *$ \\
PAM & $79(72-87)$ & & $84(68,5-92)$ & $0,70 *$ \\
PAS & & & & 0,810 \\
FR & $124,6 \pm 24$ & $119 \pm 28,8$ & & 0,670 \\
\hline
\end{tabular}

Legenda: FC: Frequência Cardíaca; PAS: Pressão Arterial Sistólica; PAD: Pressão Arterial Diastólica; PAM: Pressão Arterial Média; FR: Frequência Respiratória; DP: Desvio Padrão; IIQ: Intervalo Interquartil; *Teste Mann-Whitney; $\square$ Teste T-student 


\section{Discussão}

Segundo os dados coletados neste estudo, a NMES não resultou em alterações hemodinâmicas significativas e não houveram ajustes dos medicamentos vasoativos em pacientes críticos, tornando seu uso seguro nesta população. Sendo assim, a depender da fase crítica em que o paciente se encontre - seguindo os preceitos de segurança da mobilização precoce pode-se iniciar um programa de atividades de contrações musculares visando a minimização de danos causados pelo imobilismo $4,7,13,14$.

Buscando uma estratégia intervencionista precoce, Akar et al. ${ }^{10}$ utilizaram a NMES associada a exercícios ativos em pneumopatas como mobilização precoce e identificaram redução da FC neste grupo, sugerindo redução do trabalho cardíaco proporcionado pela NMES. Em contrapartida, Gerovasili et al5 encontraram aumento de FC e PAS significativas em pacientes críticos, atribuindo essas alterações à ativação de ergoreflexos e metaboreflexo, gerando aumento de carga simpática que, por sua vez, resulta em alterações na resistência vascular e trabalho cardíaco. Contudo, em seu estudo foi utilizado uma relação de trabalho de 12 segundos de contração para 6 segundos de relaxamento proporcionando um menor tempo de repouso com maior chance de fadiga muscular e alteração do fluxo de microcirculação ${ }^{8}$.

Em outro estudo, Segers et al. ${ }^{3}$ buscaram analisar alterações hemodinâmicas e a qualidade da contração muscular através daNMES em pacientes com determinados níveis de edema e uso ou não de vasopressores. Estes autores não encontraram alterações hemodinâmicas significativas e sugeriram que a presença de edema, vasopressores e estados pró inflamatórios, como a sepse, pode impactar negativamente na contração muscular.

Os estados pró inflamatórios podem proporcionar efeitos de hipermetabolismo que desencadeia um aumento do catabolismo proteico, resultando em sobrecarga cardíaca e renal ${ }^{15}$. Porém, em estudos anteriores 5,16 houve reduções nos níveis de citocinas inflamatórias e aumento na liberação de células endoteliais progenitoras - potencializadoras da microcirculação e regeneração tecidual - reafirmando os benefícios do exercício através da NMES. Mesmo observando as vantagens na aplicação, é necessário seguir os critérios de segurança na mobilização precoce dessa população crítica ${ }^{11,12}$ para que os efeitos positivos auxiliem na recuperação dos pacientes.

Os resultados deste estudo são limitados pelo número pequeno da amostra, mas pode ser bem tolerado visto que se trata de uma série de casos, que servirá de base para a realização de um estudo maior. No entanto percebe-se a necessidade de existir na literatura mais estudos sobre a temática a fim de influenciar programas de mobilização precoce.

\section{Conclusão}

Em suma foi possível evidenciar nesta pesquisa que a aplicação da eletroestimulação neuromuscular no doente crítico, desde que respeitando os limites estabelecidos e parâmetros corretos baseados em evidências, é uma técnica segura e viável na UTI, visto que não foi observado diferenças estatisticamente significantes em relação às variáveis hemodinâmicas estudadas.

\section{Contribuições dos autores}

Pinto DS foi responsável pela redação, delineação do método, coleta e organização de dados e revisão de literatura. Duarte HB foi responsável pela revisão crítica do conteúdo, delimitação do método, coleta, organização e tratamento dos dados e revisão de literatura. Costta CA foi resposável pela revisão de literatura, e coleta e organização dos dados. Anjos JLM foi responsável pela revisão crítica do conteúdo, organização e tratamento dos dados e orientação do projeto. Gaspar LC e Melo RL participaram da coleta e da revisão de literatura. Menezes CS foi responsável pela delineação do método, revisão de literatura e revisão crítica do manuscrito.

\section{Conflitos de interesses}

Nenhum conflito financeiro, legal ou político envolvendo terceiros (governo, empresas e fundações privadas, etc.) foi declarado para nenhum aspecto do trabalho submetido (incluindo mas não limitando-se a subvenções e financiamentos, participação em conselho consultivo, desenho de estudo, preparação de manuscrito, análise estatística, etc.). 


\section{Referências}

1. Lipshutz AKM, Gropper MA. Acquired Neuromuscular Weakness and Early Mobilization in the Intensive Care Unit. Anesthesiology. 2013;118(1):202-15. doi: 10.1097/ALN.0b013e31826be693

2. Puthucheary Z, Harridge S, Hart N. Skeletal muscle dysfunction in critical care: Wasting, weakness, and rehabilitation strategies.

Crit Care Med. 2010;38(10 Suppl):S676-82. doi: 10.1097/ cCM.0b013e3181f2458d

3. Segers J, Hermans G, Bruyninckx F, Meyfroidt G, Langer D, Gosselink R. Feasibility of neuromuscular electrical stimulation in critically ill patients. J Crit Care. 2014;29(6):1082-8. doi: 10.1016/j. jcrc.2014.06.024

4. Gerovasili V, Stefanidis K, Vitzilaios K, Karatzanos E, Politis P, Koroneos $\mathrm{A}$ et al. Electrical muscle stimulation preserves the muscle mass of critically ill patients: a randomized study. Crit Care 2009;13(5):R161. doi: $10.1186 /$ cc8123

5. Gerovasili V, Tripodaki E, Karatzanos E, Pitsolis T, Markaki V, Zervakis D et al. Short-term Systemic Effect of Electrical Muscle Stimulation in Critically III Patients. Chest 2009;136(5):1249-1256. doi: $10.1378 /$ chest.08-2888

6. Xu C, Kou Y, Zhang P, Han N, Yin X, Deng J et al. Electrical Stimulation Promotes Regeneration of Defective Peripheral Nerves after Delayed Repair Intervals Lasting under One Month. PLoS One. 2014;9(9):e105045. doi: 10.1371/journal.pone.0105045

7. Sillen MJH, Franssen FME, Gosker HR, Wouters EFM, Spruit MA. Metabolic and Structural Changes in Lower-Limb Skeletal Muscle Following Neuromuscular Electrical Stimulation: A Systematic Review. PLoS ONE. 2013;8(9):e69391. doi: 10.1371/journal. pone.0069391

8. Brum PC, Forjaz CLM, Tinucci T, Negrão CE. Adaptações agudas e crônicas do exercício físico no sistema cardiovascular. Rev Paul Educ Fís. 2004;18:21-31.

9. Strasser EM, Stättner S, Karner J, Klimpfinger M, Freynhofer M, Zaller V et al. Neuromuscular Electrical Stimulation Reduces Skeletal Muscle Protein Degradation and Stimulates Insulin-Like Growth Factors in an Age- and Current-Dependent Manner: A Randomized, Controlled Clinical Trial in Major Abdominal Surgical Patients. Ann Surg. 2009;249(5):738-43. doi: 10.1097/ SLA.0b013e3181a38e71

10. Akar O, Günay E, Sarinc Ulasli S, Ulasli AM, Kacar E, Sariaydin M et al. Efficacy of neuromuscular electrical stimulation in patients with COPD followed in intensive care unit: Rehabilitation in intensive care unit. Clin Respir J. 2017;11(6):743-50. doi: 10.1111/ crj. 12411
11. Hickmann CE, Castanares-Zapatero D, Bialais E, Dugernier J, Tordeur A, Colmant L et al. Teamwork enables high level of early mobilization in critically ill patients. Ann Intensive Care. 2016;6(1):80. doi: 10.1186/s13613-016-0184-y

12. Adler J, Malone D. Early mobilization in the intensive care unit: a systematic review. Cardiopulm Phys Ther J. 2012;23(1):5-13.

13. Hodgson CL, Stiller K, Needham DM, Tipping CJ, Harrold M, Baldwin CE et al. Expert consensus and recommendations on safety criteria for active mobilization of mechanically ventilated critically ill adults. Crit Care. 2014;18(6):658. doi: 10.1186/s13054014-0658-y

14. Burtin C, Clerckx B, Robbeets C, Ferdinande P, Langer D, Troosters $\mathrm{T}$ et al. Early exercise in critically ill patients enhances short-term functional recovery. Crit Care Med. 2009;37(9):2499505. doi: 10.1097/CCM.0b013e3181a38937

15. Sachetti A, Carpes MF, Dias AS, Sbruzzi G. Safety of neuromuscular electrical stimulation among critically ill patients: systematic review. Rev Bras Ter Intensiva. 2018;30(2):219-225. doi: 10.5935/0103-507X.20180036

16. Stefanou C, Karatzanos E, Mitsiou G, Psarra K, Angelopoulos E, Dimopoulos $S$ et al. Neuromuscular electrical stimulation acutely mobilizes endothelial progenitor cells in critically ill patients with sepsis. Ann. Intensive Care. 2016;6(1):21. doi: 10.1186/s13613016-0123-y 